\title{
Age-related changes in the passive resisting moments at the hip
}

\author{
Masaru HIGA*, Kazumasa MATSUDA*, Fusako KAWABATA*, Masayoshi ABO* \\ and Satoshi KAKUNAI* \\ *Department of Mechanical Engineering, University of Hyogo \\ Shosha 2167, Himeji, Hyogo 671-2280, Japan \\ E-mail: higa@eng.u-hyogo.ac.jp
}

Received 27 June 2013

\begin{abstract}
The purpose of this study was to investigate age-related changes in the passive resisting moments at the hip. The changes in the passive resisting moments were hypothesized to occur due to age-related changes in muscle stiffness. Two groups of healthy men participated in this study: young men (approximately 24 years of age) and old men (approximately 68 years of age). Subjects were positioned in a left lateral decubitus position with their left limb supported on a table. With a subject relaxed, an experimenter slowly moved the subject's limb by pulling or pushing the handle attached to the lower limb via a load cell. The subject's hip was moved in passive range of flexion-extension motions. The joint kinematics, measured by a motion capture system, and load cell readings were used to compute the passive resisting moments-joint angle curves at the hip. The passive resisting moments at the hip were found to considerably depend on the adjacent knee angle. The maximal resisting moments, when the hip was extended, were larger for the older group than for the younger group. The measured resisting moments were finally validated against the resisting moments calculated by a musculoskeletal computer model for the same movements. The computer model was able to predict measured tendencies of the moments at the hip. However, further adjustments of the computer model are required to represent the aging effects precisely.
\end{abstract}

Keywords : Aging, Hip, Passive moment, Joint stiffness, Musculoskeletal computer model

\section{Introduction}

In The alterations that take place in adult muscles are of interest to physical therapists, occupational therapist, gerontologists, orthopedic surgeons, and other health care providers. It has been demonstrated that older muscles exhibit a substantial loss of isometric maximum force, prolonged twitch contraction, and increased passive elastic stiffness(Hasson and Caldwell, 2012; Ochala et al., 2007; Thelen, 2003) . Further, the range of joint motion decreases with advancing age (Brown et al., 1999; Roach and Miles, 1991; Thelen, 2003). An increase in muscle elastic stiffness due to aging is possibly associated with the decline in the range of joint motion that occurs with advancing age (Rosant et al., 2007).

While several groups have studied the passive resisting joint moments of single joints (Riener and Edrich, 1999; Silder et al., 2007), few groups have studied the change of the moments that occurs with advancing age and/or disease (Gajdosik et al., 1999). In order to distinguish between pathological and physiological changes in aging muscle and to gain knowledge that would be valuable for rehabilitation programs for older people, it is necessary to understand the aging process in terms of changes in skeletal muscle structure and function. Hence, the purpose of this study was to measure the passive resisting moments over a wide range of motion at the hip and the knee, taking into account the influence of aging. On the basis of these experimental results, a mathematical approach is also proposed that can calculate the same moment assuming the experimental conditions. 


\section{Materials and Methods 2.1 Subjects}

Subjects in this study included five younger men and four older men (Table 1). A statistical analysis indicated that their height and weight did not differ among groups ( $p>0.3)$. Their body mass index (BMI) did not differ among groups $(p>0.1)$. Subjects with "Underweight" $($ BMI $<18.5)$ and "Obese" (BMI>30.0), those are the classification criteria of world health organization (WHO) (Consultation, 2004), were excluded from this study beforehand. Although physical activities varied both within and among the groups, all subjects reported their routine physical activities such as walking, running, swimming or playing some sports. Based on the study by Gajdosik (Gajdosik et al., 1999), activity levels were rated as extremely active, very active, fairly active or minimally active and non-active. Subjects with extremely active and non-active were also excluded from this study beforehand. All subjects gave written informed consent for the measurements of passive resisting moment at the hip. All subjects were healthy without serious past illnesses, musculoskeletal trauma or persistent joint pain in their lower limb, and they were in good physical condition at the time that measurements were taken.

Table 1 Mean (s.d.) age, mass, and height of the nine subjects tested.

\begin{tabular}{|c|c|c|c|c|}
\hline & Number of subjects & Age [year] & Mass [kg] & Height [cm] \\
\hline Youngers & 5 & $23.8(0.84)$ & $64.8(2.22)$ & $170.0(5.35)$ \\
\hline Olders & 4 & $67.5(2.38)$ & $61.8(8.42)$ & $165.3(7.37)$ \\
\hline
\end{tabular}

\subsection{Measurements of passive resisting moment}

The experimental apparatuses used in this study are shown in Fig. 1. A subject was positioned left lateral decubitus position on a horizontal bench constraining the pelvis against motion by a lumbosacral support and two plungers clamped against the anterior superior iliac spines. The subject's left lower limb was supported against gravity on a flat table via a low-friction cart placed under the medial side of the lower limb. All tests were performed using the left lower limb. The subject's knee was also fixed at each desired angle (0, 30, 60 and 90 degrees) by a custom knee brace. A strain gauge instrumented uni-axial load cell (TCLN-5KNA, Tokyo Sokki Kenkyujo Co., Ltd., Japan) with a handle was attached to the thigh located distally to the hip joint. Outputs of the load cell were recorded by a digital data logger (NR-500 ST-04, KEYENCE Co., Ltd. Japan). Three-dimensional position and orientation of the lower limb were tracked by a motion capture system with markers placed on palpable anatomical landmarks (Micron Tracker2, Claron Technology, Canada). Both the motion capture system and the dater logger were connected to a personal computer, enabling synchronization of all data.

With the subject relaxed, the hip angles and force histories were recorded while an experimenter slowly moved the subject's limb by pulling or pushing the handle attached to the load cell. Each subject's hip was moved in passive range of flexion-extension motions while continuously measuring the hip joint kinematics. Although the range of motion was supposed to be from 0 to 90 degree in flexion, the operator stopped moving then went back to the other direction when a subject expressed any pain or discomfort. These movements were slow enough so that the viscous and inertial components were negligible as compared to the elastic component. The passive resisting flexor moments were calculated from the moment arms (distance from the hip center to the load cell) and applied forces by the operator. Five cycles of movements were taken at each knee angle, making a total of 20 individual cycles.

\subsection{Numerical calculation of the passive resisting moment}

In order to validate the experimental measurements, a musculoskeletal three-dimensional lower limb with surrounding muscles model was developed using a three-dimensional computer aided design (CAD) software (Fig. 2). A variety of resources were used in this model. A straight line model of muscle-tendon actuators was adopted and bone geometries were based upon a commercially available human bone replica (1301-170; Pacific Research Labs, Vashon Island, WA). As for the bone replica, bone geometry is not meant to model the average human pelvis, but only to model one generic human pelvis (183 cm Male Caucasian). Muscle attachment data and parameters were derived from 
literatures (Arnold et al., 2010; Delp et al., 1990; Delp and Zajac, 1992; Dostal and Andrews, 1981; Zajac, 1989). The age of their values is not uniform because they came from various specimens. Other details of this model can be seen in the literature (Higa et al., 2011).

External moments around the hip joint were applied to move the lower limb in the same movements as in the experiments constraining the knee at each angle (0, 20,60 and 90 degrees). Assuming all muscle excitations were zero, the resisting moments around the hip were calculated. The resisting moments were assumed to be equal and opposite to the internal moments calculated from the muscle forces. The calculation of the internal moments, based on vector summations, was very simple without muscle excitations.

\section{Results}

Regardless of the knee angles, the older group exhibited higher values of the resisting moments at the hip when the hip was extended. Conversely the younger group exhibited smaller values of the resisting moments (larger in negative value) when the hip was flexed. And the older group also exhibited the smaller values of the range of motion in flexion. Mean values of these moments against the hip flexion angles are plotted (Fig. 3 and 4). Positive values in the flexor moments indicate the moments resisting extension at the hip and negative values reflect the moments resisting flexion at the hip. The resisting moments at the hip joint were dependent on the position of the knee joint over a wide range of flexion-extension movements at the hip. Flexing a subject's knee consistently increased the moments resisting
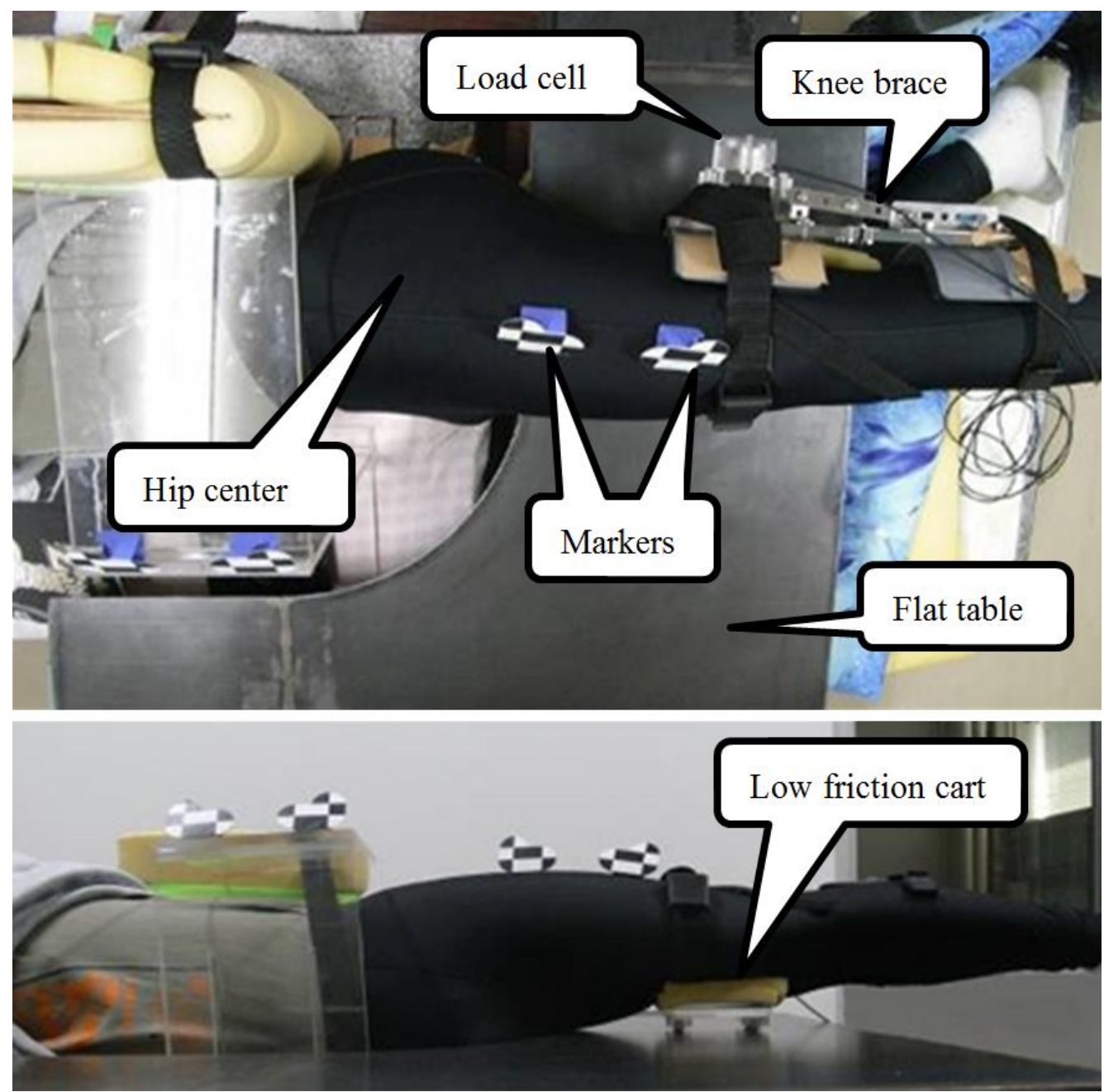

Figure 1 These two pictures show experimental set-up and a subject viewed from above (top) and from horizon (bottom). The subject lay in a left lateral decubitus position with the knee was fixed at 0 degrees in flexion in these pictures. Every apparatus required to measure (e.g., the knee brace, the load cell, reflection markers and the low friction cart) were mounted. 


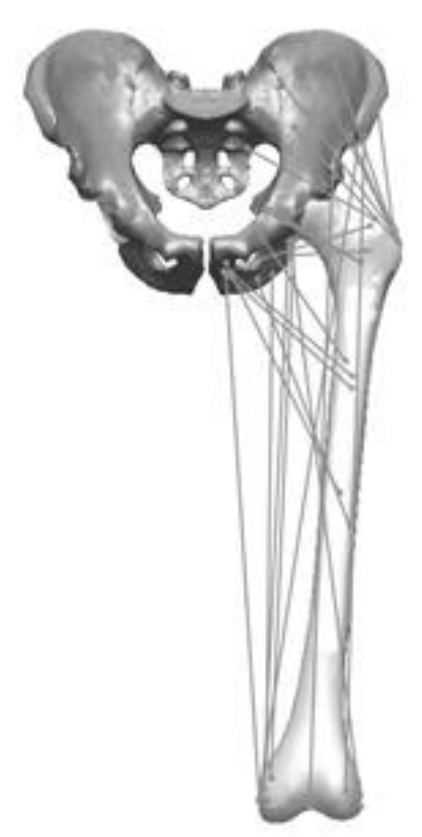

Figure 2 Three-dimensional musculoskeletal computer model of the hip was developed by a computer aided design (CAD). Lines of action for muscle tendon units and the bone geometries are shown.

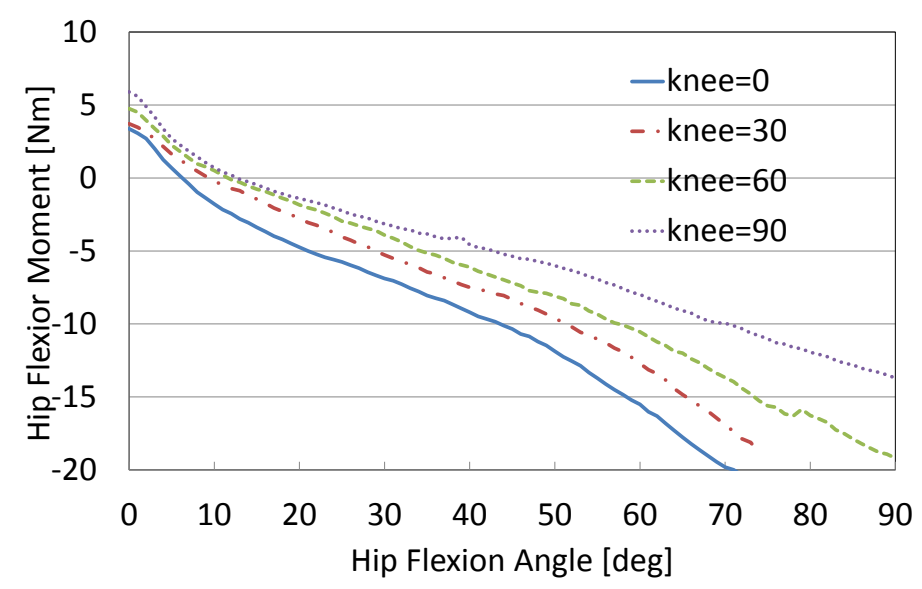

Figure 3 Mean values of passive resisting moments for younger subjects at various knee angle positions are plotted against the hip flexion angles.

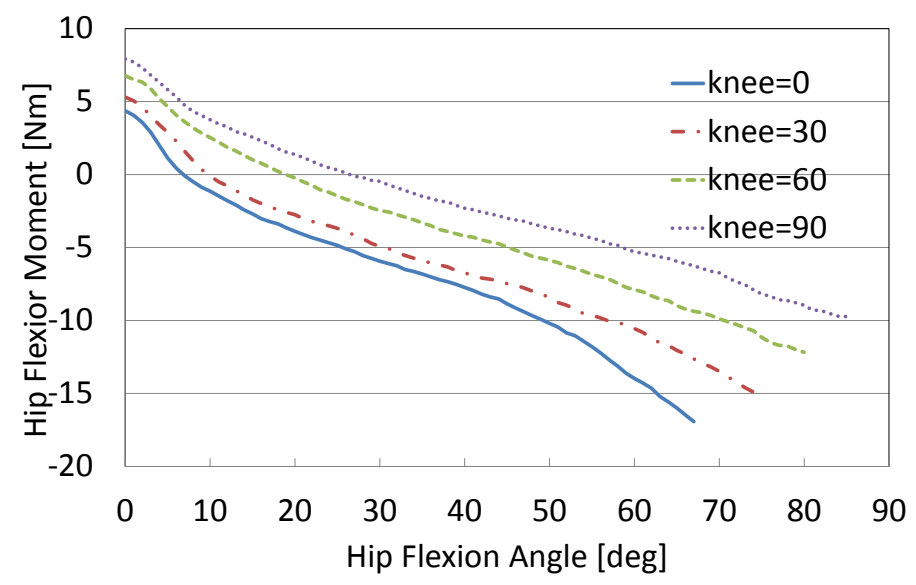

Figure 4 Mean values of passive resisting moments for older subjects at various knee angle positions are plotted against the hip flexion angles. 
extension at the hip. Conversely extending the knee increased the moments resisting flexion at the hip. Two-joint muscles crossing the hip and the knee had a consistent effect on this tendency. For example, with the knee set in the maximum extended position, hamstring muscles were stretched and tend to extend the hip. Some curves are finished before 90 degree in flexion where the lowest range of motion of a subject was considered. The peak values of the moments and the range of motions are listed in table 2. Although differences of the moments between the older group and the younger group were not significant at every hip joint angles, higher flexor moments (higher extension resistance) occurred in the older group when the hip was extended regardless of the knee angles, while lower flexor moments (higher flexion resistance) occurred in the younger group when in high hip flexion angles regardless of the knee angles. Some of the subjects showed more than 90 degree of the range of motion (indicated by + signs in table1). Hence, statistical analyses of the range of motion on these configurations were not conducted. To clear repeatability of measurements, five times measurements of the flexor moments on a representative older subject against hip flexion angles are plotted (Fig. 5). The repeated trials produced qualitatively similar results within a subject.

The flexor moments calculated from the computer model showed similar tendencies to the measured moments. However, some discrepancies were observed. For example, the calculated moments were considerably smaller (larger in negative value) than the measured moments when the hip was flexed with the knee was extended $(\mathrm{knee}=0$, hip $=90)$. And the moments when the hip was extended with the knee was flexed (knee=90, hip=0) were also somewhat higher than the measured moments (Fig. 6).

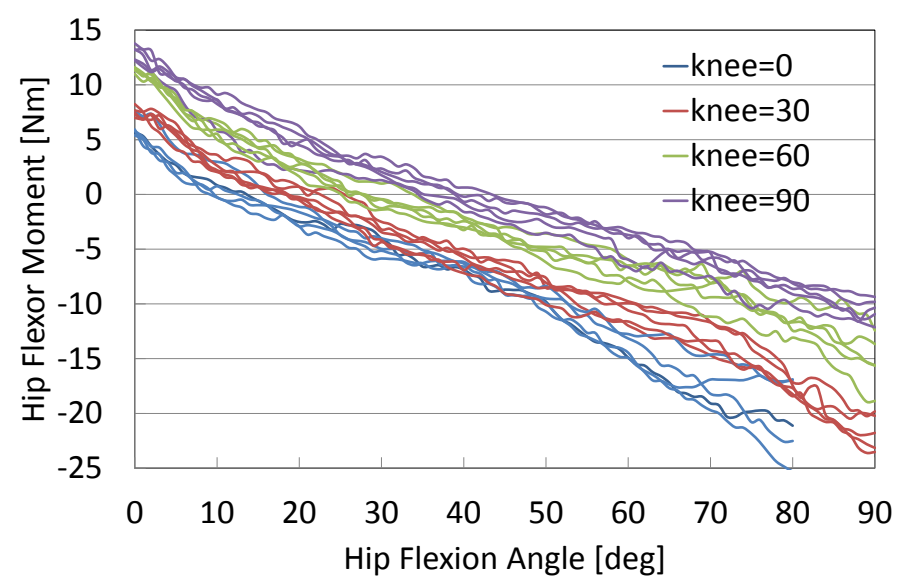

Figure 5 Passive resisting moments of five repetitive measurements for a representative older subject with various knee angles of flexion are plotted against the hip flexion angles.

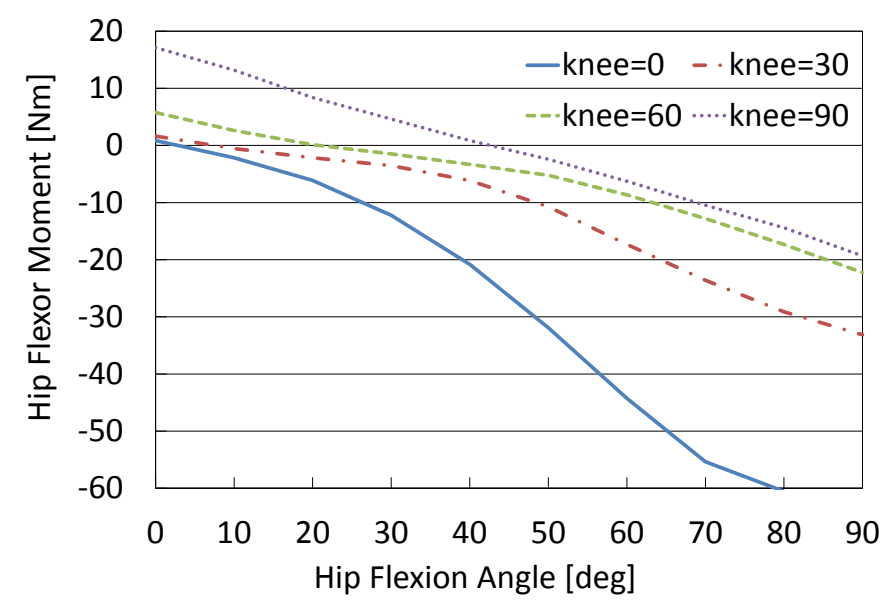

Figure 6 Calculated passive resisting moments at the hip with various knee angles are plotted against the hip flexion angles. 


\section{Discussion}

This study demonstrated a correlation between the passive resisting moments at the hip and advancing age. The influence of the knee joint angle on the passive resisting moments at the hip are attributed to the moment induced by two joint muscles. The observed correlation between the passive resisting moments at the hip and the knee joint is consistent with the previous reports (Riener and Edrich, 1999; Silder et al., 2007). The hip flexor moments were the largest (moments resisting extension at the hip) with the knee was flexed in 90 degrees. This was induced due to the stretching of some of the muscles at the anterior compartment of the thigh by flexing the knee. The hip flexor moments were the smallest (moments resisting flexion at the hip were high) with the knee fully extended. This was due to the stretching of the hamstring muscles by extending the knee.

Our comparison between the two groups of measurements demonstrated that the older group exhibited higher values of the flexor moments at the hip when the hip was extended. Conversely, the younger group exhibited smaller values of the flexor moments (higher in extensor moments) when the hip was flexed. Thelen reported how to adjust muscle parameters to simulate older adults muscle in a computer model. Thelen said that the passive muscle strain due to maximum isometric force should be reduced from 0.6 for young adults to 0.5 for older adults to account for the relative increase in passive stiffness (Thelen, 2003). However, if this adjustment was applied to our computer model, only higher absolute values of resisting moments would be calculated in the older model. That is not enough to express the unique tendencies observed in our measurements. In another interesting example of animal experiments; older rats showed substantially increased flexor muscle stiffness (measured during extension) and unchanged of extensors in the ankle joint (Wolfarth et al., 1997). Of course, having suggested that this kind of unique change of ankle muscle stiffness due to aging, this change of muscle parameter doesn't always apply to other joints.

Some limitations of this study must also be addressed. First, this study contained a small number of subjects, which limits the statistical power of analysis. The difference of the flexor moment at the hip between these two groups was statistically significant only in one configuration (hip $=60^{\circ}$, knee $=90^{\circ}$ ), although the mean value of the flexor moments showed consistent tendency between the two groups. These observations suggest that additional data points

Table 1 Peak resisting moments at the hip and the range of motions derived from the moment-flexion curves are listed. The peak resisting moments were selected when the hip in 0 degrees and 60 degrees in flexion. "+" signs mean some of the subjects exhibited over 90 degrees of the range of motions in flexion.

* Significant at $\mathrm{p}<0.05$.

\begin{tabular}{|c|c|c|c|c|}
\hline & Hip angle $\left[{ }^{\circ}\right]$ & Knee angle $\left[{ }^{\circ}\right]$ & Younger & Older \\
\hline \multirow{8}{*}{$\begin{array}{l}\text { Hip flexor moments } \\
{[\mathrm{Nm}]}\end{array}$} & \multirow[t]{4}{*}{0} & 0 & 3.37 & 4.36 \\
\hline & & 30 & 3.71 & 5.30 \\
\hline & & 60 & 4.75 & 6.77 \\
\hline & & 90 & 5.90 & 7.91 \\
\hline & \multirow[t]{4}{*}{60} & 0 & -15.51 & -13.97 \\
\hline & & 30 & -12.71 & -10.55 \\
\hline & & 60 & -10.53 & -7.89 \\
\hline & & 90 & -7.98 & -5.29 \\
\hline \multirow{4}{*}{$\begin{array}{l}\text { Range of motion in } \\
\text { flexion }\left[{ }^{\circ}\right]\end{array}$} & & 0 & 77.0 & 69.0 \\
\hline & & 30 & $86.8+$ & 78.5 \\
\hline & & 60 & $87.6+$ & $85.5+$ \\
\hline & & 90 & $90+$ & $88.75+$ \\
\hline
\end{tabular}


are required to provide appropriate statistical analysis. Second, the computer model gave good predictions of experimental measurements, but was less accurate in some cases. There are some discrepancies between measured and calculated moments at the hip. The computer model was based on a male Caucasian who is taller than the averages of our subjects' heights. Hence, the use of this model is fundamental to roughly ensure the cross-validation of experimental measurements in vitro by means of numerical calculation. Some improvements of the model especially subject-specific model development just like the other study (Hasson and Caldwell, 2012) is also desirable. In this study, however, it was difficult to obtain bone geometries using an X-ray apparatus from healthy subjects. Third, it was impossible to estimate the differences of the range of motion statistically between two groups in some joint configurations, because the measurements were limited at 90 degree in flexion though some subjects showed over 90 degree of the range of motion. However, to measure the range of motion was not the first purpose of this study and it had been consented to the every subjects that the measurements never accompany any pain or discomfort. Hence, extreme flexion angles were not tried.

In this study, we demonstrated that the hip moments are different between the older group and the younger group. This finding suggests that the flexor muscles at the hip in the older adults might be stiffer than those in the younger adults. Hence, it seems that uniform increases of the muscle stiffness to simulate the older adult muscles may not be proper way.

\section{References}

Arnold, E.M., Ward, S.R., Lieber, R.L., Delp, S.L., A model of the lower limb for analysis of human movement, Annals of biomedical engineering 38, (2010), pp.269-279.

Brown, M., Fisher, J.S., Salsich, G., Stiffness and muscle function with age and reduced muscle use, Journal of orthopaedic research : official publication of the Orthopaedic Research Society 17, (1999), pp.409-414.

Consultation, W.H.O.E., Appropriate body-mass index for Asian populations and its implications for policy and intervention strategies, Lancet 363, (2004), pp.157-163.

Delp, S.L., Loan, J.P., Hoy, M.G., Zajac, F.E., Topp, E.L., Rosen, J.M., An interactive graphics-based model of the lower extremity to study orthopaedic surgical procedures, IEEE transactions on bio-medical engineering 37 , (1990), pp.757-767.

Delp, S.L., Zajac, F.E., Force- and moment-generating capacity of lower-extremity muscles before and after tendon lengthening, Clinical orthopaedics and related research, (1992), pp.247-259.

Dostal, W.F., Andrews, J.G., A three-dimensional biomechanical model of hip musculature, Journal of biomechanics 14, (1981), pp.803-812.

Gajdosik, R.L., Vander Linden, D.W., Williams, A.K., Influence of age on length and passive elastic stiffness characteristics of the calf muscle-tendon unit of women, Physical therapy 79, (1999), pp.827-838.

Hasson, C.J., Caldwell, G.E., Effects of age on mechanical properties of dorsiflexor and plantarflexor muscles, Annals of biomedical engineering 40, (2012), pp.1088-1101.

Higa, M., Tanino, H., Abo, M., Kakunai, S., Banks, S.A., Effect of acetabular component anteversion on dislocation mechanisms in total hip arthroplasty, Journal of biomechanics 44, (2011), pp.1810-1813.

Ochala, J., Frontera, W.R., Dorer, D.J., Van Hoecke, J., Krivickas, L.S., Single skeletal muscle fiber elastic and contractile characteristics in young and older men, The journals of gerontology, Series A, Biological sciences and medical sciences 62, (2007), pp.375-381.

Riener, R., Edrich, T., Identification of passive elastic joint moments in the lower extremities, Journal of biomechanics 32, (1999), pp.539-544.

Roach, K.E., Miles, T.P., Normal hip and knee active range of motion: the relationship to age, Physical therapy 71, (1991), pp.656-665.

Rosant, C., Nagel, M.D., Perot, C., Aging affects passive stiffness and spindle function of the rat soleus muscle, Experimental gerontology 42, (2007), pp.301-308.

Silder, A., Whittington, B., Heiderscheit, B., Thelen, D.G., Identification of passive elastic joint moment-angle relationships in the lower extremity, Journal of biomechanics 40, (2007), pp.2628-2635.

Thelen, D.G., Adjustment of Muscle Mechanics Model Parameters to Simulate Dynamic Contractions in Older Adults, Journal of biomechanical engineering 125, (2003), pp.70-77.

Wolfarth, S., Lorenc-Koci, E., Schulze, G., Ossowska, K., Kaminska, A., Coper, H., Age-related muscle stiffness: predominance of non-reflex factors, Neuroscience 79, (1997), pp.617-628.

Zajac, F.E., Muscle and tendon: properties, models, scaling, and application to biomechanics and motor control, Critical reviews in biomedical engineering 17, (1989), pp.359-411. 